\title{
MILK ANALYSIS.
}

By Dr. Bennett F. Davenport.*

IN the discussion of the relative merits of the different methods of milk analysis which was published in the June number of The ANALYST, the many advantages of a simple modification of Wanklyn's method does not, I think, receive its due consideration. This modification, which I have adopted, I have already published in my Annual Report as Milk Inspector for the City of Boston, Mass., 1885, and as Analyst to the Massachusettis State Board of Health in their Annual Report for 1886, page 138. As being now the method generally followed by most of the official milk analysts in New England, a simple description of it may be of interest to others.

The 5 grms. of milk are weighed off in a large flat-bottom platinum capsule of full $2 \frac{1}{2}$ inches diameter on the bottom, and about 3 inches across the top. The $\frac{1}{2}$-inch high side turns up from the bottom, not with a sharp angle, but slight rounding, this being about the curve with which the milk runs up the rim of the capsule drawn by capillarity. The milk in drying down does not thus form any thicker deposit at the angle of the side than elsewhere upon the about 5 square inches of bottom surface of the capsule. This relatively large amount of surface, one square inch to each grm. of sample taken, causes each inch to be covered with only a little over a single grain of dried milk solids. The deposit is therefore so very thin as to be readily exhausted of its fat in its subsequent treatment with boiling petroleum naphtha. When using such small dishes as were originally proposed by Wanklyn, the residue would be so thick, that is about three times, as would naturally render any such rapid method of extraction as I employ impossible, as the English analysts have learned. Each capsule has its serial number engraved upon it, and they are made to weigh a little over 25 grms. each, that the bottoms may be stiff enough to remain perfectly flat-a matter of very great importance. They are also made to differ from each other only in the second and third places of decimals, so that only those weights have to be changed in many weighings, which is a matter of no little saving of labour where many score of weighings are to be made each day. A table of their weights is kept within the case of the scales. These capsules, containing their samples, are placed upon a constant-level closed-top water-bath, of the peculiar construction described on page 269 of the Journal of Analytical Chemistry. This will hold twenty-five capsules at a time. Here, being surrounded by atmosphere not already nearly saturated with moisture, as would be the case if they were upon a water-bath with openings on its top, they quickly evaporate to apparent dryness. They are then transferred for their final drying to a constant weight to one of Weisnegg's large porcelain-lined air-baths, regulated to the constant temperature of $105^{\circ} \mathrm{C}$. Here they are dried in about half an hour, when they are cooled in a desiccator and each weighed immediately upon being taken out, to guard against their rapid gain in weight from exposure to the air. Replaced upon the closed-top water-bath, the capsule is filled from a wash bottle with petroleum naphtha, of the quality of the benzine of the U. S. Pharmacopeia, revision of 1880 . This, unlike ether, will dissolve out neither milk-sugar nor lactic acid from the dried milk-solid residue. Moreover, one gallon of it costs but as much as one pint of ether would, which is a matter of some little con- 
sequence to one like me, who has had to use about 100 gallons of it in milk analyses during the past five years.

The naphtha in the capsule, after being allowed to boil down about one-half, is decanted off against a rod into a basin to guard against the remote possibility of some flakes of milk residue being poured off with it. Replaced upon the bath, the capsule is refilled with naphtha. This boiling up and decanting off is repeated three to four times, when after the last one the outside of the capsule is washed off with naphtha played upon it from the wash-bottle, to prevent any residue of fat being left there. The capsule, then finally replaced upon the bath to dry off the naphtha, is then cooled and weighed as before, the butter being rather determined from the loss of weight in the solids than by the weight of the evaporated washings.

The ash is made by ignition of the capsule over a one-inch wide Bunsen lamp, which thus gives so wide a flame as not to require a heating to a high temperature at one point, and thus a possible loss by the volatilisation of the potassium chloride. The milk-sugar I determine by the use of a Soleil-Ventzke saccharimeter, in the same manner as has now been several times described in the journals by Drs. Wiley, Vieth, and others. The other ingredients which reduce copper in Fehling's solution, are not thus reckoned as sugar, by which the apparent amount of albuminoids present, which are obtained by difference, would be diminished.

The peculiarities of my modification is then the use of an evaporating capsule of such a very large relative area as will leave the residue thin enough to be readily exhausted by the boiling naphtha. Thus a diameter of $2 \frac{1}{2}$ inches in the capsule will do for 5 grms. of better than average quality of milk, with a surety that there will not be as much as one-tenth of 1 per cent. of fat left as a maximum error. And surely this is fine enough for all commercial work, and it is much to be doubted if much which affects to be more accurate by a decimal than this is really so. The method, as involving no transfer of substance, cannot well be made more simple for the determination of total solids, fat and ash. When the average life of such an evaporating capsule under reasonable usage is considered, it cannot well be cheapened, although the outlay for a kilo. of platinum for forty capsules like mine does, it is true, involve an original expense of nearly 400 dollars. The shape of my capsules makes possible completeness of extraction with the solvent used, and the construction of the closed water-bath hastens the process in no small degree. 